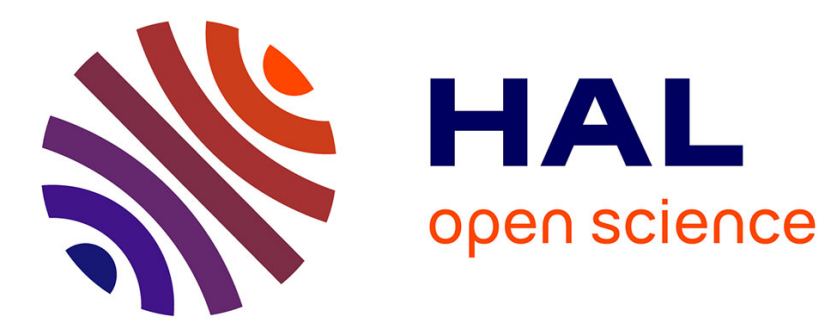

\title{
Localisation de particules par chambre à étincelles
}

O. Beer, C. Laspalles, Y. Terrien, G. Vallois

\section{To cite this version:}

O. Beer, C. Laspalles, Y. Terrien, G. Vallois. Localisation de particules par chambre à étincelles. Revue de Physique Appliquée, 1969, 4 (2), pp.305-306. 10.1051/rphysap:0196900402030501 . jpa-00243274

\section{HAL Id: jpa-00243274 https://hal.science/jpa-00243274}

Submitted on 1 Jan 1969

HAL is a multi-disciplinary open access archive for the deposit and dissemination of scientific research documents, whether they are published or not. The documents may come from teaching and research institutions in France or abroad, or from public or private research centers.
L'archive ouverte pluridisciplinaire HAL, est destinée au dépôt et à la diffusion de documents scientifiques de niveau recherche, publiés ou non, émanant des établissements d'enseignement et de recherche français ou étrangers, des laboratoires publics ou privés. 


\title{
LOGALISATION DE PARTIGULES PAR GHAMBRE A ÉTINGELLES
}

\author{
O. BEER, G. LASPALLES, Y. TERRIEN et G. VALLOIS, \\ Service de Physique Nucléaire à Moyenne Énergie, C.E.N., Saclay.
}

Résumé. - Le système de détection de particules par chambre à étincelles utilisé au cyclotron de Saclay est décrit. La haute tension n'est appliquée que pour des particules de type donné, et tout déclenchement est automatiquement interdit pendant le temps mort de la chambre. La localisation par partage de courant est améliorée par la normalisation du signal de localisation par la charge totale traversant la chambre.

Abstract. - The spark-chamber particle detection system used at the Saclay cyclotron is described. The high voltage is applied only for the type of particle desired. Any triggering is automatically forbidden during the dead time of the chamber. The localisation by current division is improved by the normalization of the localisation signal by the total charge which flows across the chamber.

Pour étudier les réactions nucléaires avec une très bonne résolution, tout en pouvant accéder aux résultats dès leur acquisition, nous utilisons un spectromètre magnétique sur la focale duquel les particules sont localisées par une chambre à étincelles déjà partielle- ment décrite [1] et dont nous rapportons ici les améliorations.

C'est seulement lors du passage de certaines particules dans la chambre que la haute tension est appliquée entre les deux grilles. Pour cela, les signaux 
recueillis par deux photomultiplicateurs associés à un cristal traversé par ces particules sont traités par un circuit logique (fig. 1) qui permet de ne déclencher la haute tension que dans des conditions prédéterminées. Les signaux issus des deux photomultiplicateurs sont additionnés dans un mélangeur. Le sélecteur de bande permet de ne garder que les signaux correspon-

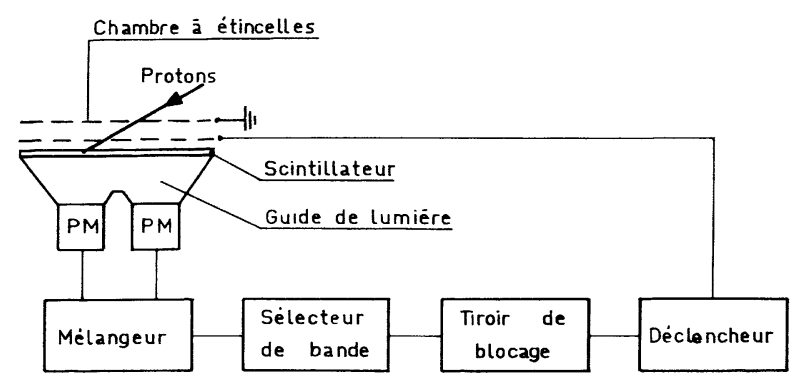

FIG. 1. - Schéma logique du dispositif de déclenchement.

dant à un type de particule donné. Après un déclenchement, pour permettre au gaz de la chambre de revenir à son état initial, le tiroir de blocage coupe le circuit logique pendant un temps lié au temps mort de la chambre à étincelles. Le déclencheur envoie sur la chambre une impulsion de haute tension $(10 \mathrm{kV} / \mathrm{cm}$ environ) à travers une capacité qui se décharge par la formation d'une étincelle entre les deux grilles. Le temps de montée de cette impulsion est de 50 ns. Le délai entre le passage d'une particule dans la chambre à étincelles et l'application de la haute tension sur celle-ci est de $200 \mathrm{~ns}$, ce qui est inférieur au temps de mémoire de la chambre.

La chambre à étincelles comprend essentiellement deux grilles parallèles placées dans un gaz, constituées par des fils de tungstène de diamètre $30 \mu \mathrm{m}$, espacés de $0,5 \mathrm{~mm}$. Le gaz est de l'hélium à la pression atmosphérique, circulant à faible débit $\left(0,3 \mathrm{~cm}^{3} / \mathrm{s}\right.$ environ). L'écart entre les deux grilles est de $4 \mathrm{~mm}$. Les dimensions utiles de la chambre sont $45 \mathrm{~cm}$ sur $1,6 \mathrm{~cm}$.

Lorsqu'une étincelle s'est produite dans la chambre, il subsiste, dans le gaz, des ions, des électrons et des atomes métastables qui peuvent provoquer une nouvelle étincelle si une haute tension est appliquée entre les grilles, sans qu'une nouvelle ionisation primaire ait eu lieu. La durée pendant laquelle ce phénomène peut se produire est le temps mort de la chambre. Pour le mesurer, on applique à la chambre, après le passage d'une particule, deux impulsions de haute tension séparées l'une de l'autre par un délai connu, et on mesure la probabilité d'obtenir une étincelle (étincelle parasite) avec la deuxième impulsion. Nous appelons temps mort la valeur du délai pour lequel cette probabilité est $10 \%$. Si le blocage est égal au temps mort ainsi défini, et si le taux de comptage moyen est de $100 \mathrm{cps} / \mathrm{s}$, ceci nous conduit à un nombre d'étincelles parasites inférieur à $1 \%$ du nombre total d'étincelles. Le temps mort est évidemment d'autant plus grand que la haute tension appliquée à la chambre est plus grande; pour le réduire, on applique en permanence un champ de balayage de 100 à $300 \mathrm{~V} / \mathrm{cm}$. La valeur du temps mort ainsi obtenu est inférieure à $3 \mathrm{~ms}$.

L'étincelle est localisée par la mesure du partage du courant qu'elle transporte [2]. Pour éviter l'erreur due à la fluctuation de ce courant, qui provient de la décharge de la capacité du déclencheur (voir plus haut), un calculateur PDP 8 en ligne effectue la division du signal de localisation par un signal proportionnel à ce courant. La figure 2 montre le même spectre avec et sans division. Le signal de localisation est fonction linéaire de la position de l'étincelle sur la chambre à $\pm 2 \%$ près. La résolution spatiale obtenue est de l'ordre de $0,7 \mathrm{~mm}$, ce qui conduit à une résolution inférieure à $20 \mathrm{keV}$ pour la réaction indiquée sur la figure 2 .

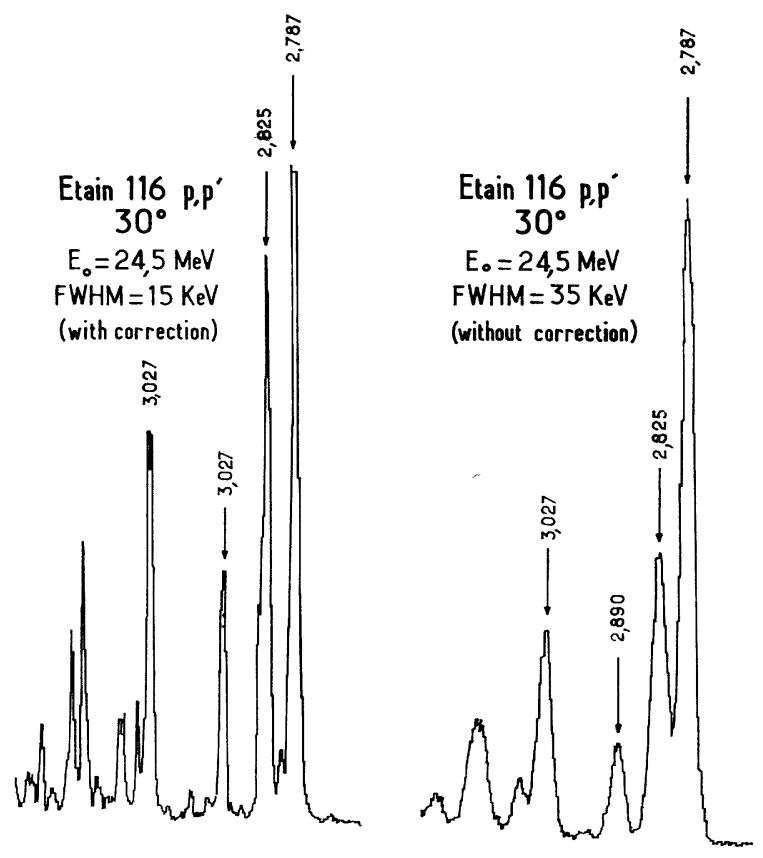

FIG. 2. - Spectres avec et sans division.

\section{BIBLIOGRAPHIE}

[1] Saudinos (J.), Valiois (G.) et Laspalles (C.), Nucl. Instr. Methods, 1967, 46, 229.
[2] Charpak (G.), Massonet (L.) et Favier (J.), Nucl. Instr. Methods, 1963, 24, 501. 